\title{
Development of a motion platform for an educational flight simulator
}

\author{
Albert S. J. Van Heerden, \\ Department of Mechanical and Aeronautical Engineering, University of Pretoria. Present address: Denel \\ Dynamics, P.O. Box 7412, Centurion 0046, South Africa.
}

Raine Lidbetter, Leon Liebenberg (corresponding author), and Edward H. Mathews, Center for Research and Continued Engineering Development, North-West University (Pretoria campus), Suite no. 93, Private Bag X30, Lynnwood Ridge 0040, South Africa. Consultants to TEMM Intl. (Pty) Ltd.

Josua P. Meyer,
Department of Mechanical and Aeronautical Engineering, University of Pretoria, Pretoria 0002, South Africa.

E-mail: LLiebenberg@researchtoolbox.com 
Abstract Flight simulators are regularly used in undergraduate and postgraduate training of mechanical and aeronautical engineers. Due to advances in computing technology, several flight simulation-related tasks can now be accomplished in real-time using low-cost PC platforms and inexpensive commercial software. The difficulty in realizing an educational flight simulator system with motion platform therefore lies with the design and construction of an effective motion platform. Costs become exorbitant when simulation platforms of more than two degrees-of-freedom (i.e., pitch and roll) are attempted. This paper describes the development of a motion drive system to facilitate a two degrees-of-freedom (pitch and roll) motion platform for use in undergraduate engineering training. Use was made of off-the-shelf PC equipment and flight simulation software and hardware, together with commercial actuators and drive systems. The motion platform was manufactured from square-tubing and consisted of three frames; the stationary main frame and, rotating inside this, the roll frame and pitch frame, respectively. These rotate relative to each other and were actuated by two similarly-sized DC motors and gearbox/chain transmissions. The system effectively simulates the pitch and roll motions of commercial airliners, using a low-cost, easy maintainable motion platform. The educational value of the simulator was two-fold: first, it is to be displayed in the science exploratorium (SciEnza) of the University of Pretoria and second, the simulator provides a platform on which mechanical (as well as electrical, electronic and computer) engineering students could conduct practical work in courses such as dynamics and control, and on which final-year and postgraduate students could conduct research.

Keywords: educational flight simulator; motion platform; two degrees-of-freedom 


\section{Nomenclature}

Abbreviations

CG Centre of gravity

CNC Computer numerical control (machining)

DC Direct current

DOF Degree of freedom

FSUIPC Flight Simulator Universal Inter-Process Communication

FSX Microsoft Flight Simulator X

NACoE National Aerospace Centre

PID

Proportional integral and differential (compensator)

Roman letter symbols

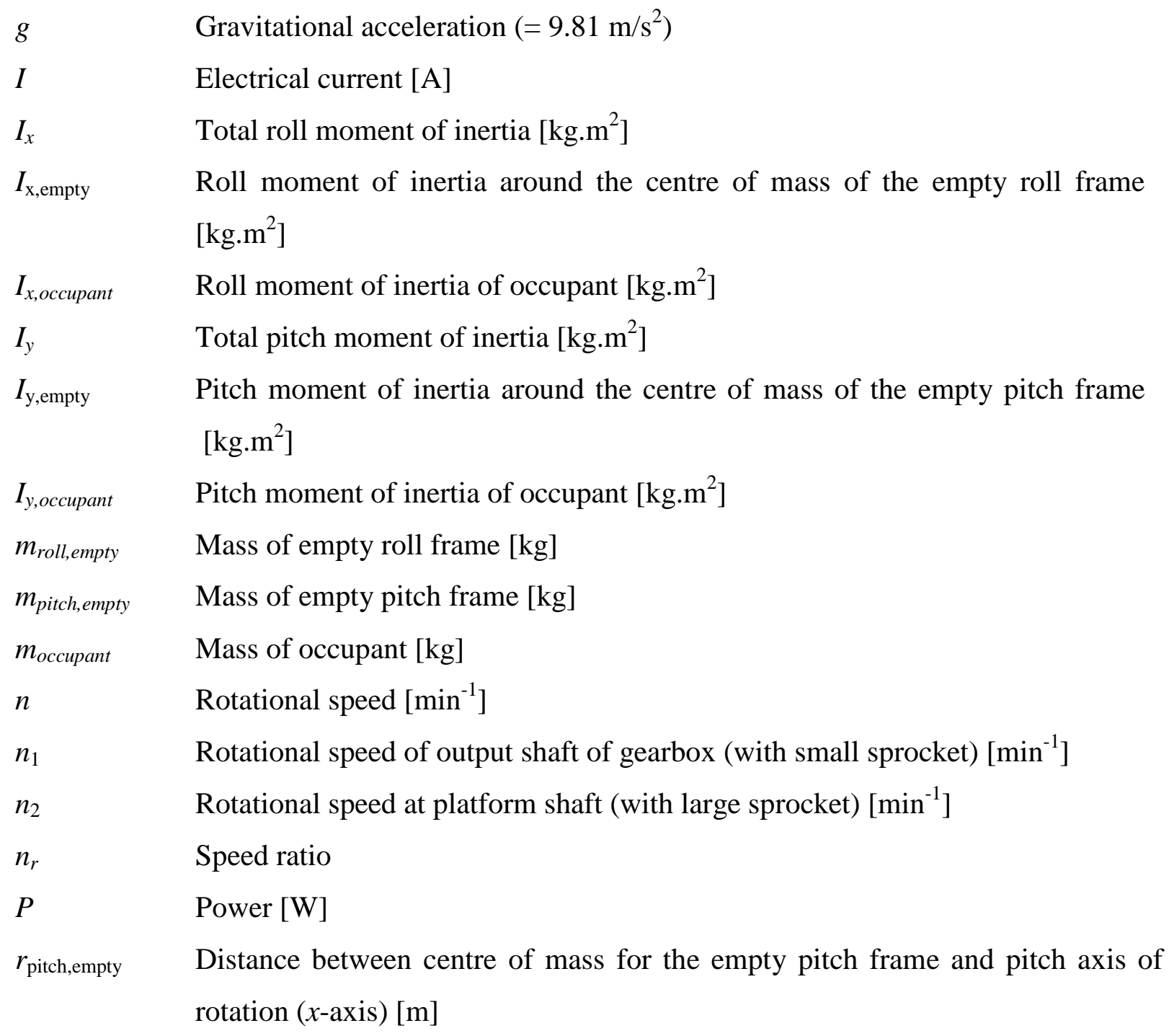




$\begin{array}{ll}r_{\text {pitch,occupant }} & \text { Distance (in the } x \text {-Z plane) between the centre of mass of occupant and the } \\ & \text { centre of the pitch axis [m] } \\ r_{\text {off }} & \text { Distance between CG of occupant in forward position and axis of rotation [m] } \\ r_{\text {roll,empty }} & \text { Distance between centre of mass for the empty roll frame and roll axis of } \\ & \text { rotation ( } x \text {-axis) [m] } \\ r_{\text {roll,occupant }} & \text { Distance (in the } x \text {-Z plane) between the centre of mass of occupant and the } \\ T_{\text {acc, } x} & \text { centre of the roll axis [m] } \\ T_{a c c, y} & \text { Torque [N.m] } \\ T_{g e a r b o x, o u t} & \text { Torque required to create angular acceleration of the roll platform [N.m] } \\ T_{i n} & \text { Torque at gearbox output shaft [N.m] } \\ T_{\max } & \text { Maximum torque required [N.m] } \\ T_{\max , x} & \text { Maximum torque for roll [N.m] } \\ T_{\max , y} & \text { Maximum torque for pitch [N.m] } \\ T_{\text {offset }, y} & \text { Torque to overcome a maximum estimated static offset of the centre of mass } \\ V & \text { of the occupant with respect to the axis of rotation [N.m] } \\ & \text { Voltage [V] }\end{array}$

Greek letter symbols

$\begin{array}{ll}\alpha_{\mathrm{x}} & \text { Angular acceleration around } x \text {-axis (roll/bank) }\left[\mathrm{rad} / \mathrm{s}^{2}\right] \\ \alpha_{\mathrm{y}} & \text { Angular acceleration around } y \text {-axis (pitch) }\left[\mathrm{rad} / \mathrm{s}^{2}\right] \\ \eta_{\text {gearbox }} & \text { Gearbox efficiency } \\ \omega_{\mathrm{m}} & \text { Motor rotational speed }[\mathrm{rad} / \mathrm{s}] \\ \omega_{\mathrm{max}} & \text { Maximum angular velocity }[\mathrm{rad} / \mathrm{s}] \\ \omega_{\mathrm{x}} & \text { Angular velocity around } x \text {-axis (roll/bank) }[\mathrm{rad} / \mathrm{s}] \\ \omega_{\mathrm{y}} & \text { Angular velocity around } y \text {-axis (pitch) }[\mathrm{rad} / \mathrm{s}]\end{array}$




\section{Introduction}

Many universities make use of flight simulators in the training of mechanical and aeronautical engineers, or in other disciplines [1-5]. The simulators are integrated into undergraduate and graduate courses, such as introduction to aerodynamics, flight mechanics, aircraft design, aircraft propulsion, automatic control systems and flight dynamics and controls. However, flight simulators that have four or more degrees-of-freedom are usually prohibitively expensive and complex.

A flight simulator usually consists of seven subsystems, namely simulation models, cockpit instruments, visual-cueing system, motion-cueing system, audio-cueing system, instructor station, cockpit controls, and force feedback system [6,7]. The relationship between these different subsystems is illustrated in Fig. 1.

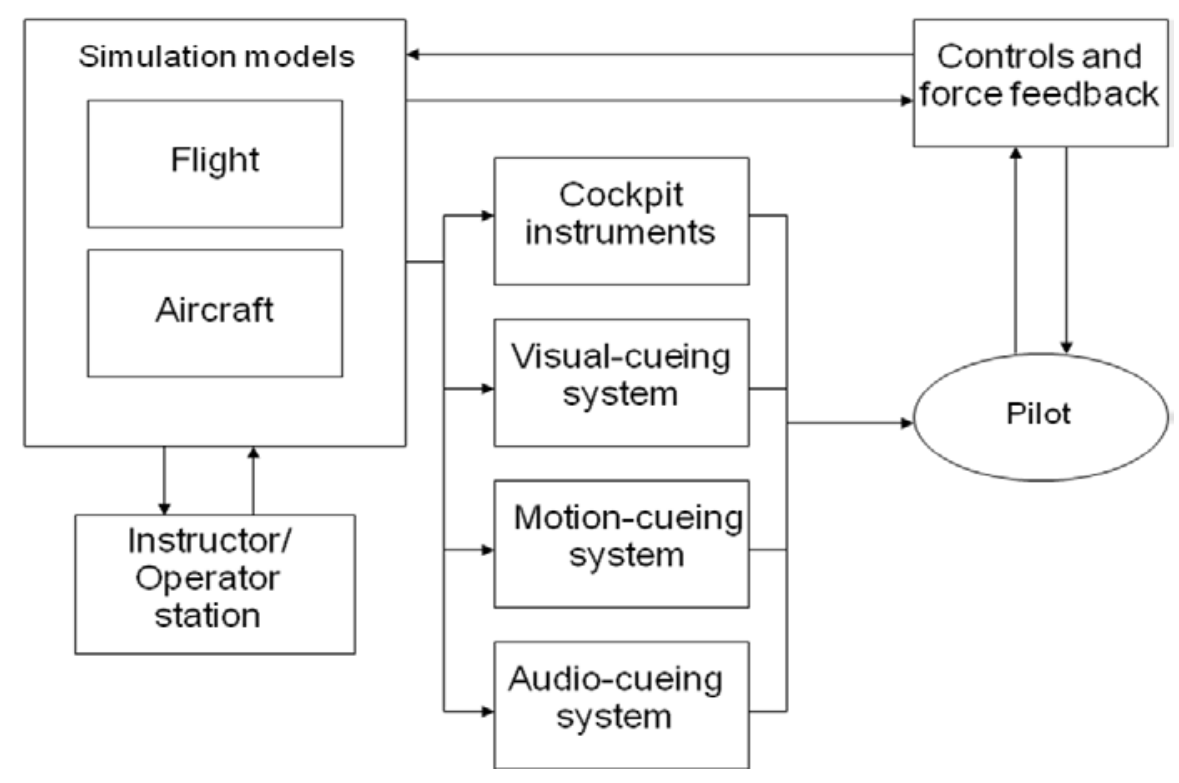

Fig. 1 A generic flight simulation functional model (adapted from $[6,8]$ )

The simulation model is central to any flight simulator system. This software program is divided into two parts: an aircraft model and a flight model. The aircraft model simulates an aircraft's aerodynamic characteristics, dynamics, flight controls, engine, navigation, auto- 
flight and fuel systems. The flight model simulates the environment in which the aircraft model is operating [6].

Flight simulators essentially present the user with the illusion that he or she is actually flying in an aircraft. This is mainly achieved through a set of 'cues', or hints, which are produced in response to control inputs provided by the user. These cues usually include visual, audio and vestibular types [7,9].

For the present project, the flight and aircraft models, as well as the cockpit instruments, and audio- and visual-cueing systems were provided by low-cost commercial simulation software. The audio cues were provided through speakers and the visual cueing from PC monitors. There were no instructor/operator stations as these functions are performed by the pilot.

The vast progress in computer technology has made available ample computing performance to accomplish several flight simulation-related tasks, including real-time simulation of complex dynamic models and sophisticated visual scene generation on standard low-cost PC platforms $[6,10,11]$. With affordable computer hardware and software available, the only remaining item to be resolved to allow for low-cost, pilot-and hardware-in-the-loop simulation for educational purposes, is the motion platform.

The motion platform provides translational and rotational motion cues. Hydraulic actuators are typically used on high-end motion platforms [2,12], usually affording the motion platform with four or more degrees-of-freedom. This results in versatile, but extremely costly solutions, which certainly are not a consideration for use in the average academic establishment. 
This paper describes the development of a motion drive system to facilitate a two degrees-offreedom (pitch and roll) motion platform for a flight simulator being used in the Department of Mechanical and Aeronautical Engineering at the University of Pretoria. The main aim of the project is to exploit off-the-shelf PC equipment and flight simulation software and hardware, together with standard actuators and drive systems, to generate a low-cost, easy maintainable educational flight simulator.

\section{The motion-cueing system}

A functional diagram of a typical flight simulator motion-cueing system is shown in Fig. 2. Note that no station for an instructor was required for this project, as the developed simulator was not a flight-training simulator, but rather a flight-awareness simulator.

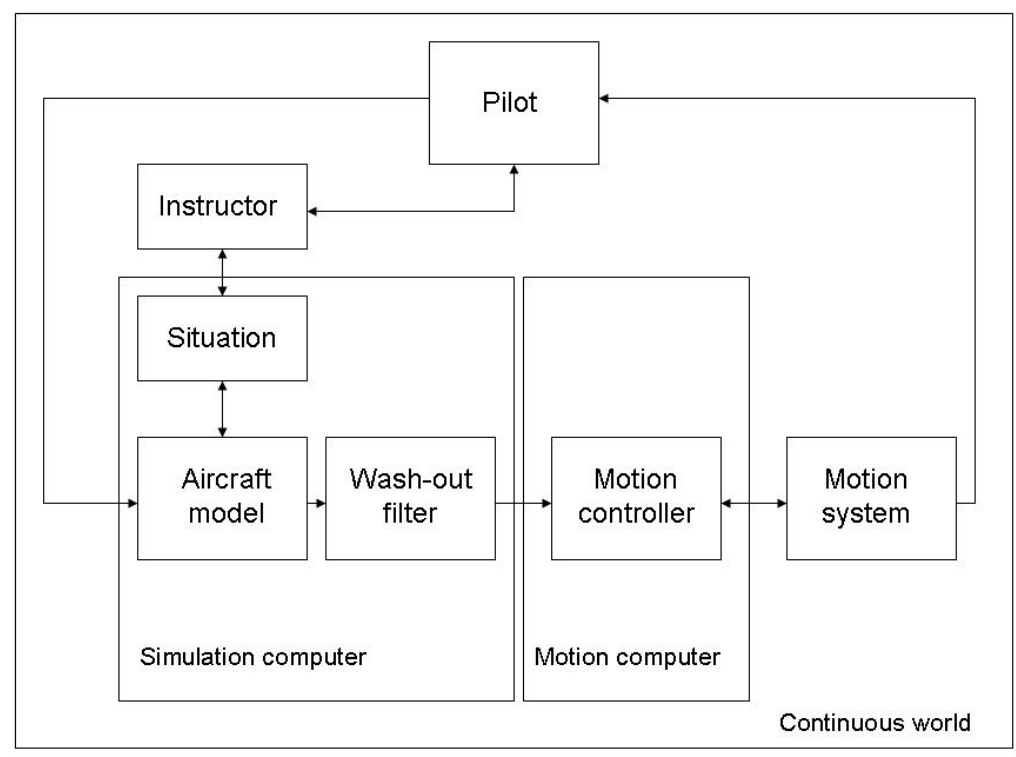

Fig. 2 Motion simulator (adapted from [13])

The user provides the aircraft model with a specific input. This is then processed by the simulation model, which provides an appropriate output to the so-called wash-out filters (also known as motion-cueing algorithms, $[7,14]$ ), which, in turn, give motion commands to the 
motion controller [12]. Wash-out filters are part of the motion algorithms and are not discussed in detail here, as a commercial software package, named BFF Motion Driver v. 2 [15], was employed.

The motion controller, which essentially consists of motion control software and a signal processor, translates and processes the motion information from the motion-cueing algorithms, as well as motion feedback from potentiometers, or speed encoders, and then issues commands to the motion system (the motion actuators and platform) in the form of electrical signals. The signals activate the actuators to replicate the motion cueing and translate the motion to the simulator platform. The pilot then senses the motion of the platform and observes the other outputs from the visual and audio cues.

Simulators become increasingly expensive with the number of degrees-of-freedom achieved. Therefore, to avoid a highly expensive and complex mechanical system, it was decided to choose only two degrees-of-freedom; namely pitch and roll. These are strongly associated with motions of flight, can be integrated well with flight simulation software, and are the most critical of the six degrees-of-freedom in terms of motion-sensing for a human $[11,16-$ $18]$.

\section{Motion platform: structure}

\section{Primary design requirements}

The flight simulator was initially earmarked for use in the University of Pretoria's science exploratorium (SciEnza) for school students. The simulator had to be housed in a space with dimensions of $3 \mathrm{~m} \times 3 \mathrm{~m} \times 3 \mathrm{~m}$. It was decided to limit the mass of the occupant (the 'pilot') to that of a $95^{\text {th }}$ percentile male, which was $97.7 \mathrm{~kg}[19]$. 


\section{Platform design}

To achieve the separate motions of pitch and roll, the design of the platform required three components; a stationary base, a roll frame and a pitch frame. These components were assembled in a cascading manner with one frame inside the other. It was decided that the movement in the degrees-of-freedom were to be controlled using two individual actuators one for roll and one for pitch.

The frames were manufactured from mild steel by means of CNC tube bending. The material was chosen for its good strength-to-weight ratio and manufacturability, and low cost. It provided an ample framework to support equipment, the occupant and the forces applied to it.

The square tubing also provided surface area for the mounting of pillow blocks/ bearing housings, safety panels, monitors, input controls and actuators, while being aesthetically pleasing. Figure 3 illustrates the flight simulator platform and the axis system used in all calculations (the origin of this system is at the imaginary intersection between the two axes of rotation), while TABLE 1 shows some of the salient characteristics of the platform. 
TABLE 1 Salient characteristics of the motion platform (with no occupant)

\begin{tabular}{|l|l|}
\hline Characteristic & Value \\
\hline Steel square tubing sizes & $25 \times 25 \times 2.5 \mathrm{~mm}$ \\
\hline Shaft (axes of rotation) diameters & $25 \mathrm{~mm}$ \\
\hline Roll frame (with pitch frame) mass $-\mathrm{m}_{\text {roll,empty }}$ & $110.9 \mathrm{~kg}$ \\
\hline Pitch frame mass $-\mathrm{m}_{\text {pitch,empty }}$ & $90.4 \mathrm{~kg}$ \\
\hline $\begin{array}{l}\text { Distance between centre of mass for roll frame and roll axis of rotation }(x \text {-axis })- \\
r_{\text {roll,empty }}(\text { Estimated using a solid model of the platform) }\end{array}$ & $0.05 \mathrm{~m}$ \\
\hline $\begin{array}{l}\text { Distance between centre of mass for pitch frame and pitch axis of rotation }(y \text {-axis) } \\
-r_{\text {pitch,empty }} \text { (Estimated using a solid model of the platform) }\end{array}$ & $0.155 \mathrm{~m}$ \\
\hline $\begin{array}{l}\text { Roll moment of inertia (around the centre of mass of the roll frame) }-I_{x, \text { empty }} \\
\text { (Estimated using a solid model of the platform) }\end{array}$ & $16.93 \mathrm{~kg} \cdot \mathrm{m}^{2}$ \\
\hline $\begin{array}{l}\text { Pitch moment of inertia (around the centre of mass of the pitch frame) }-I_{\mathrm{y}, \text { empty }} \\
\text { (Estimated using a solid model of the platform) }\end{array}$ & $29.34 \mathrm{~kg} \cdot \mathrm{m}^{2}$ \\
\hline
\end{tabular}


(a)

(b)
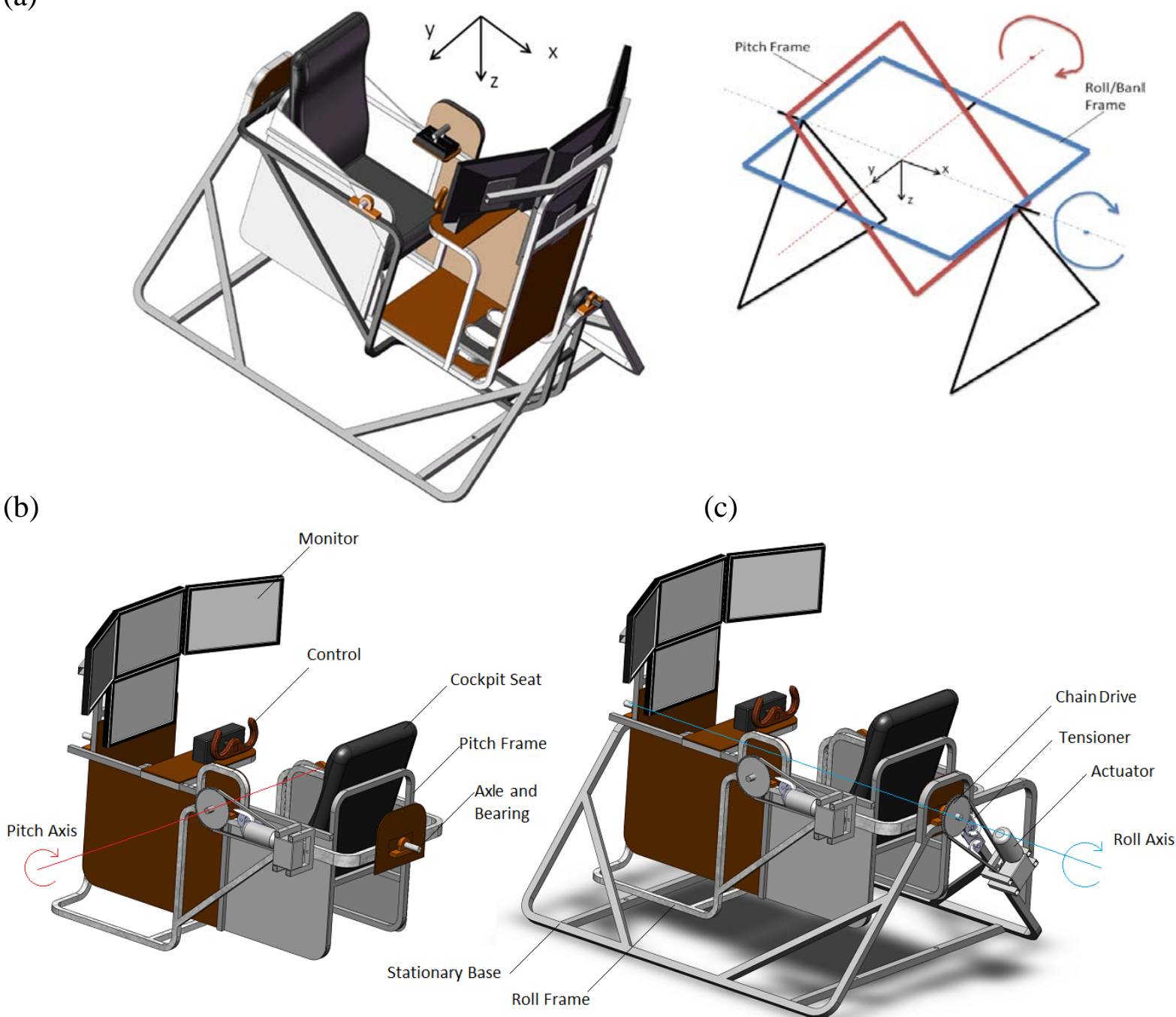

Fig. 3 Solid model showing layout of the platform structure: (a) the complete system, (b) the pitch axis, and (c) the roll axis

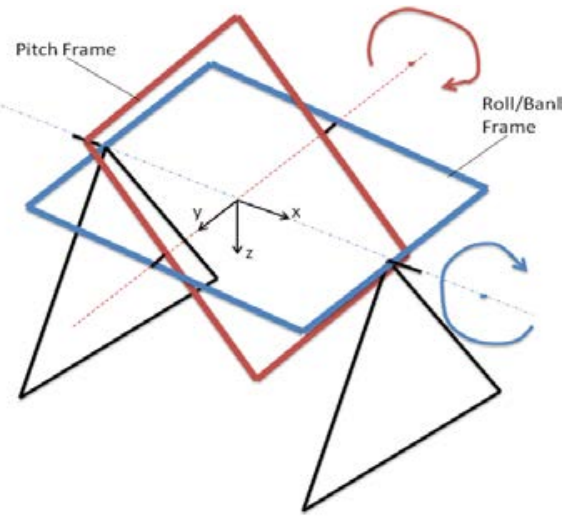

(c) 


\section{Simulated aircraft and simulation software}

An inexpensive, non-FAA-certified (Federal Aviation Administration), commercial off-theshelf package was selected, namely the popular Microsoft Flight Simulator X, [5,20]. The software was operated on a PC with four monitors (visual cues) and two speakers (audio cues). A control column, throttle quadrant and rudder pedals were also used. The specifications of the PC that was used can be viewed in TABLE 2.

TABLE 2 PC specifications

\begin{tabular}{|l|l|}
\hline Component & Specification \\
\hline Processor & Intel-P Core 2 Duo, 2.8 GHz \\
\hline RAM & $2048 \mathrm{MB}$ \\
\hline Hard disk space & $160 \mathrm{~GB}$ \\
\hline Operating system & Microsoft Windows XP Professional \\
\hline Audio output & Creative SBS A200 Speaker System \\
\hline Display output & Four 19" LCD monitors \\
\hline Graphics & $\begin{array}{l}\text { Matrox TripleHead2Go external multi-display adapter and Nvidia GeForce GTS 250 } \\
\text { graphics card }\end{array}$ \\
\hline
\end{tabular}

It was decided to use a generic airliner as the aircraft to be simulated, because of the slow roll-and pitch-rates of airliners, such as the Boeing 777. This was desirable from an actuator sizing, simulator platform (structural) and safety point of view, with low-cost being the overarching goal.

\section{Actuators}

The actuators for the motion platform were selected to be brushed DC electrical motors, as they are readily available, have high starting torques, feature accurate speed and torque control (compared with AC motors), and are reliable under variable loads. Electrical motors 
represent a versatile and inexpensive solution to this type of application compared with pneumatic or hydraulic actuators.

\section{Motion control software}

The wash-out filters, together with a proportional, integral and differential (PID) controller are incorporated into a software package that was purchased from Built For Fun [15]. The software extracts the required data in real-time from the simulation model in Flight Simulator $X$, and calculates the appropriate motion cues. The data is then exported to the signal processor unit, from where it is transported to the actuator drivers. The primary data output of the software is position demand cues for the motion platform.

This software is suited for platforms where motion for the different degrees of freedom is controlled by independent motors, similar to the present project. Software settings can be altered to suit different kinds of aircraft and platforms. The values for the proportional $(\mathrm{P})$, integral (I) and differential (D) terms of the PID controller can also be changed in the software to suit individual platforms. For this project, the PID values were tuned, until satisfactory behaviour of the platform was obtained.

\section{Signal processor}

A customized signal processor unit was used, namely the 40SPU-1 card [15] that is designed for flight simulator motion platform applications. It serves as a communication bridge between the drivers (controllers), feedback and the PID software, monitors the system, and provides status updates on the system. For example, it monitors communication interruptions, stops the system in an emergency and provides continuous information on the temperature of the controllers [15]. 


\section{Actuator drivers/motor drivers}

The drivers receive position demand from the signal processing unit, amplify the current and then scale the voltage from a power supply to drive the motors. The Devantech MD03 - 24V 20A H-bridge motor drive was selected as the motor driver. It employs pulse width modulation (PWM) of the H-bridge at a frequency of $15 \mathrm{kHz}$ [21].

\section{Moments of inertia of the motion platform}

In order to estimate the torque and power required from the DC motors, as well as power transmission requirements, it was necessary to estimate the maximum mass moment of inertia. The moments of inertia for the pitch (y-axis) and roll ( $x$-axis) frames were estimated using the following equations:

For pitch:

$$
I_{y}=I_{y, \text { empty }}+I_{y, \text { occupant }}+m_{\text {pitch,empty }} r_{\text {pitch,empty }}^{2}+m_{\text {occupant }} r_{\text {pitch,occupant }}^{2}
$$

In this equation, which employs the parallel-axes theorem [22], $I_{y, \text { empty }}$ is the pitch moment of inertia of the empty pitch frame (obtained from the solid model of the frame - see TABLE $1) ; \quad I_{y, \text { occupant }}=10.25 \mathrm{~kg} \cdot \mathrm{m}^{2}$ is the moment of inertia in pitch of a $95^{\text {th }}$ percentile male around his centre of mass [19]; $m_{\text {pitch,empty }}$ is the empty mass of the pitch frame (from TABLE 1); $m_{\text {occupant }}=97.7 \mathrm{~kg}$ is the mass of a $95^{\text {th }}$ percentile male [19]; $r_{\text {pitchempty }}$ is the distance (in the $x$ $z$ plane) between the centre of mass of the empty pitch frame and the centre of the pitch shaft (from TABLE 1); and $r_{\text {pitch,occupant }}=0.166 \mathrm{~m}$ is the distance (in the $x$-z plane) between the 
centre of mass of a $95^{\text {th }}$ percentile male [19] and the centre of the pitch axis. Using (1), the pitch moment of inertia was estimated to be:

$$
\begin{aligned}
I_{y} & =I_{y, \text { empty }}+I_{y, \text { occupant }}+m_{\text {pitch,empty }} r_{\text {pitch,empty }}^{2}+m_{\text {occupant }} r_{\text {pitch,occupant }}^{2} \\
& =29.34+10.25+(90.4)(0.155)^{2}+(97.7)(0.166)^{2} \\
& =44.45 \mathrm{~kg} . \mathrm{m}^{2}
\end{aligned}
$$

Similarly, for roll:

$$
I_{x}=I_{x, \text { empty }}+I_{x, \text { occupant }}+m_{\text {roll,empty }} r_{\text {roll, empty }}^{2}+m_{\text {occupant }} r_{\text {roll, occupant }}^{2}
$$

Here $I_{x, \text { occupant }}=9.5 \mathrm{~kg} \cdot \mathrm{m}^{2}$ is the moment of inertia in roll of a $95^{\text {th }}$ percentile male around his centre of mass [19]; $r_{\text {roll,occupant }}=0.155 \mathrm{~m}$ is the distance (in the $y$-z plane) between the centre of mass of a $95^{\text {th }}$ percentile male [19] and the centre of the roll shaft. The roll moment of inertia was calculated as:

$$
I_{x}=29.05 \mathrm{~kg} \cdot \mathrm{m}^{2}
$$

Angular velocities and accelerations of a large airliner

Other parameters required to determine the required torque and power to produce platform motion were the estimated maximum angular velocities and accelerations for pitch and roll movements of a large airliner. McLafferty [23], the lead engineer at the Boeing Simulation Data Group, provided the authors with the maximum values for a generic Boeing twin-aisle airliner:

- Max. roll rate: $\omega_{\mathrm{x}}=44 \%$, or $0.768 \mathrm{rad} / \mathrm{s}$

- Max. roll acceleration: $\alpha_{\mathrm{x}}=32 \% \mathrm{~s}^{2}$, or $0.56 \mathrm{rad} / \mathrm{s}^{2}$

- Max. pitch rate: $\omega_{\mathrm{y}}=5.5 \% \mathrm{~s}$, or $0.096 \mathrm{rad} / \mathrm{s}$ 
- Max. pitch acceleration: $\alpha_{\mathrm{y}}=12 \% \mathrm{~s}^{2}$, or $0.21 \mathrm{rad} / \mathrm{s}^{2}$

These values are governed by the structural limits of the aircraft and are therefore extreme they are not actually expected to be reached during flight (and hence by the simulator). They were, however, used in the design of the drive system, as this provided for an inherent safety factor (and because of the lack of other available data). As can be seen, the maximum angular rates were for roll.

\section{Required torque and power}

The maximum estimated torque required from the drive system to provide angular movement around the axes of rotation of the roll-and pitch-platforms consists of two components (with the assumption that friction and damping torques are engineered to be negligibly low). These are the torque to create angular acceleration of the platform, and the torque to overcome a maximum estimated static offset of the centre of mass of the occupant with respect to the axis of rotation (this will happen when the occupant moves forward during the operation of the simulator). The maximum torque could therefore be estimated as follows:

\section{For pitch:}

Torque required to produce angular acceleration of the pitch platform:

$$
T_{a c c, y}=I_{y} \alpha_{y}=(44.45)(0.21)=9.33 \text { N.m }
$$

Torque to overcome a maximum estimated static offset of the centre of mass of the occupant with respect to the axis of rotation (cf. Fig. 4):

$$
T_{\text {offset }, y}=m_{\text {pitch,empty }} g r_{\text {pitch,empty }}-m_{\text {occupant }} g r_{\text {off }}
$$




$$
=(90.4)(9.81)(0.155)-(97.7)(9.81)(0.06)=79.95 \approx 80 \mathrm{~N} . \mathrm{m}
$$

The term $m_{\text {occupant }} g r_{\text {off }}$ was negative because it would cause a torque in the opposite direction than that caused by the offset of the mass of the empty pitch platform. This effect can be deduced from Fig. 4. The value of $r_{\text {off }}$ was estimated by making use of the solid model, as well as the $95^{\text {th }}$ percentile male data from Reynolds [19]. Note that it is improbable that the occupant could move further forward than this, because he will be restricted from doing so by a safety belt. Also note that occupants of lower mass than the $97.7 \mathrm{~kg}$ of a $95^{\text {th }}$ percentile male will also have the effect of causing $r_{\text {off }}$ to become smaller, which will cause more torque to be demanded from the drive system. To compensate for this, adjustable balancing weights were added to the back of the platform behind the occupant to prevent the CG from moving excessively forward.

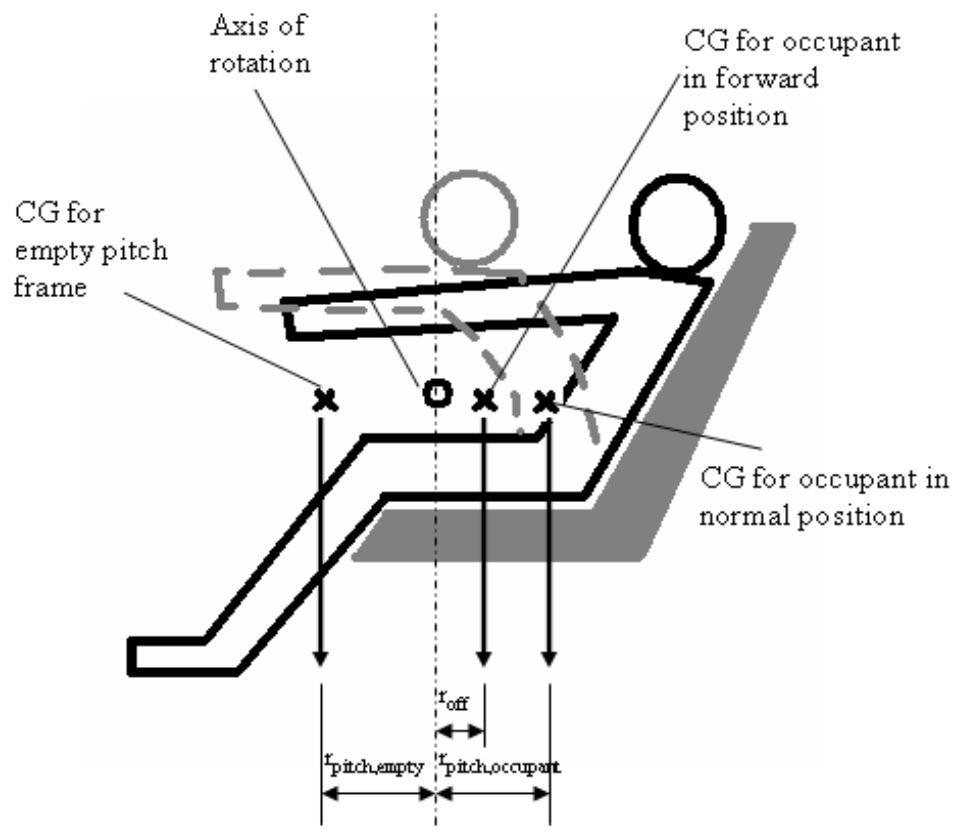

Fig. 4 CG shift due to occupant moving forward 
The maximum estimated torque required for the pitch platform was therefore calculated to be:

$$
T_{\max , y}=T_{a c c, y}+T_{\text {offset }, y}=9.33+80=89.33 \approx 90 \mathrm{~N} \cdot \mathrm{m}
$$

For roll:

No significant offset of the occupant CG was expected for the roll. The maximum torque required for roll was therefore estimated to be:

$$
T_{\text {max }, x}=T_{a c c, x}=I_{x} \alpha_{x}=(29.05)(0.56)=16.27 \text { N.m }
$$

The maximum torque required was therefore:

$$
T_{\max }=T_{\max , y}=90 \mathrm{~N} . \mathrm{m}
$$

The maximum power required (for one degree of freedom) could hence be calculated as:

$$
P_{\max }=T_{\max } \omega_{\max }=T_{\max } \omega_{x}=(90)(0.768)=69.12 \approx 70 \mathrm{~W}
$$

Maximum range of movement of the motion platform

For safety reasons, the movement of the motion platform was limited to $15^{\circ}$ for pitch and $20^{\circ}$ for roll. It is the task of the wash-out algorithms to induce the perception of movement beyond $20^{\circ}$. The platform was restricted from exceeding these positions by the motion software. Electronic limit switches were also added to stop the platform should it exceed the maximum range in an emergency. All these requirements are summarised in TABLE 3. 
TABLE 3 Actuation design requirements

\begin{tabular}{|l|l|}
\hline Requirement & Value \\
\hline Space & $3 \mathrm{~m} \times 3 \mathrm{~m} \times 3 \mathrm{~m}$ \\
\hline Maximum occupant mass & $95 \mathrm{~kg}$ \\
\hline Maximum moment of inertia & $45 \mathrm{~kg} \cdot \mathrm{m}^{2}$ \\
\hline Maximum pitch angle & $-15^{\circ}$ to $15^{\circ}$ \\
\hline Maximum roll/bank angle & $-20^{\circ}$ to $20^{\circ}$ \\
\hline Maximum torque required & $90 \mathrm{~N} \cdot \mathrm{m}$ \\
\hline Maximum power required & $70 \mathrm{~W}$ \\
\hline
\end{tabular}

\section{Actuator and transmission selection and sizing}

With the maximum torque and power requirements known, a suitable motor and transmission system could be selected. The motors had to produce rotation of the pitch-and roll-frames around their respective shafts (i.e., their axes of rotation). It will be shown that the rotational velocity would have to be reduced in order to increase the available torque from the motors, by making use of worm gearboxes and chain drives. To ensure modularity, both the pitch-and roll-drives employed the same types of motors, gearboxes and chain drives.

From equation (9), the maximum required torque of 90 N.m was calculated. Of the motors investigated, a $24 \mathrm{~V} 180 \mathrm{~W} 15 \mathrm{~A}$, permanent magnet, brushed DC motor was considered to possess the characteristics closest to the present requirements. The $70 \mathrm{~W}$ required from the motor was well below the continuous duty cycle of $180 \mathrm{~W}$ of this motor, and the risk of overheating the motors during operation was therefore low. This motor could provide a maximum continuous torque of $T_{\text {in }}=0.53 \mathrm{~N} . \mathrm{m}$ at $3000 \mathrm{~min}^{-1}$. This torque had to be increased by making use of speed reduction methods.

The available torque from the motor was increased by making use of a worm gearbox, with a speed reduction of $n_{\mathrm{r}}=100: 1$. This resulted in a maximum gearbox output speed of $30 \mathrm{~min}^{-1}$, with a mechanical efficiency of 0.53 . The output torque from the gearbox was calculated to be:

$$
T_{\text {gearbox, out }}=T_{i n} n_{r} \eta_{\text {gearbox }}=(0.57)(100)(0.53)=30.21 \mathrm{~N} . \mathrm{m}
$$


This was still too low and it was decided to use a chain drive to reduce the rotational speed even further. As stated, the maximum rotational speed at the output shaft of the gearbox was:

$$
n_{1}=30 \mathrm{~min}^{-1}
$$

And the maximum rotational speed required at the platform shaft was:

$$
n_{2}=\frac{60 \omega_{\max }}{2 \pi}=\frac{60(0.768)}{2 \pi}=7.33 \mathrm{~min}^{-1}
$$

This required a speed reduction ratio of smaller than:

$$
n_{r}=\frac{n_{1}}{n_{2}}=\frac{30}{7.33}=4.09 \approx 4
$$

The best combination of sprockets proved to be a 17-tooth sprocket for the driving sprocket (faster shaft), and a 57-tooth sprocket for the driven sprocket (connected to the shafts of the platform). The chain was a 06B Fenner chain. This combination gave a speed reduction ratio of:

$$
n_{r}=3.35
$$

These sprockets were selected based on an iterative process, with the aim of selecting sprockets with taper locks (which greatly simplified the manufacturing process). Note that this maximum output speed was slightly higher than required. The available torque for rotational movement of the platform for this speed ratio was hence determined to be:

$$
T=n_{r} T_{\text {gearbox,out }}=3.35(30.21)=101.2 \text { N.m }
$$

This torque was higher than required, which allowed for an additional inbuilt safety factor. The drive system design was therefore conservative, but ensured that none of the components would be overloaded during the operation of the system.

\section{Control System}

The platform drivers exhibited non-linear output. However, the modelling thereof was beyond the scope of this project. The non-linearities arose mainly due to the fact that the worm gearbox had low efficiency and was essentially self-locking [24]. However, the motion 
TABLE 4 Salient technical specifications

\begin{tabular}{|c|c|c|}
\hline System & 2-DOF Flight simulator with motion base & Cost (US\$) \\
\hline Simulation model & Microsoft Flight Simulator $X$ running on PC & $\begin{array}{l}\text { Donated by Microsoft } \\
\text { South Africa }\end{array}$ \\
\hline Extraction software & $\begin{array}{l}\text { FSUIPC software (to extract required data from } \\
\text { FSX) [25] }\end{array}$ & 41.21 \\
\hline Cockpit instruments & FSX instrument display on monitor & Included in PC costs \\
\hline Visual-cueing system & FSX display on three monitors & Included in PC costs \\
\hline Audio-cueing system & $2 \times$ Computer speakers & Included in PC costs \\
\hline Motion-cueing system & 2-DOF (pitch and roll) & n.a. \\
\hline Actuator type & $\begin{array}{l}2 \times \text { Transtecno EC180.240 electric DC motors } \\
(24 \mathrm{~V}, 15 \mathrm{~A} \text { Permanent magnet brushed) }[26]\end{array}$ & $\begin{array}{l}1204.13 \text { (This } \\
\text { includes the cost of } \\
\text { the gearboxes) }\end{array}$ \\
\hline Speed reduction & $\begin{array}{l}\text { 100:1 via worm gearbox, and 3.35:1 via 06B Fenner } \\
\text { chain drive, giving total reduction of } n_{r, t o t a l}=335: 1 \\
\text { per actuator }\end{array}$ & $\begin{array}{l}200.00 \text { (Only the } \\
\text { chain drives) }\end{array}$ \\
\hline Wash-out algorithms & $B F F$ motion driver software [15] & $\begin{array}{l}248.05 \text { (this includes } \\
\text { the PID software, the } \\
\text { processor unit and all } \\
\text { the supporting } \\
\text { hardware) }\end{array}$ \\
\hline Motion control software & BFF PID servo control software [15] & $\begin{array}{l}\text { Included in cost of } \\
\text { BFF motion software }\end{array}$ \\
\hline Processor unit & BFF 40SPU-1 [15] & $\begin{array}{l}\text { Included in cost of } \\
\text { BFF motion software }\end{array}$ \\
\hline Drivers & 2 x MD03 motor drivers (Robot Electronics) [21] & 156.98 \\
\hline Feedback type & Position & N.A. \\
\hline Feedback hardware & Two single-turn linear10k $\Omega$ potentiometers & 10 \\
\hline Power supply & $\begin{array}{l}\text { Dual output Adjustable 30V/20A ×2 DC Power } \\
\text { Supply Unit (PSU DP3020E) [27] }\end{array}$ & 965.72 \\
\hline Input controls & $\begin{array}{l}\text { Yoke (CH Products), throttle quadrant (CH } \\
\text { Products) and rudder pedals (CH Products) [28] }\end{array}$ & 1021.15 \\
\hline Personal computer (PC) & See Table 2 & 3161.26 \\
\hline Motion platform & $\begin{array}{l}\text { Materials, manufacturing costs, bearings, cockpit } \\
\text { seat, hardware }\end{array}$ & 450.88 \\
\hline
\end{tabular}

software allowed for the physical tuning of the P, I and D parameters of the PID controller until acceptable system behaviour was obtained.

\section{The final product}

TABLE 4 lists the salient technical specifications of the simulator. The total system cost (including PC system, platform and motion drive) was US\$7,410.00. 
Several brackets and attachments were fitted to the motion platform to accommodate the parts and components of the drive system. Brackets held the gearboxes, to which the motors were bolted, while the potentiometers and limit switches constituted the main attachments. Fig. 5 shows the assembled simulator (with chain-drive safety covers removed).

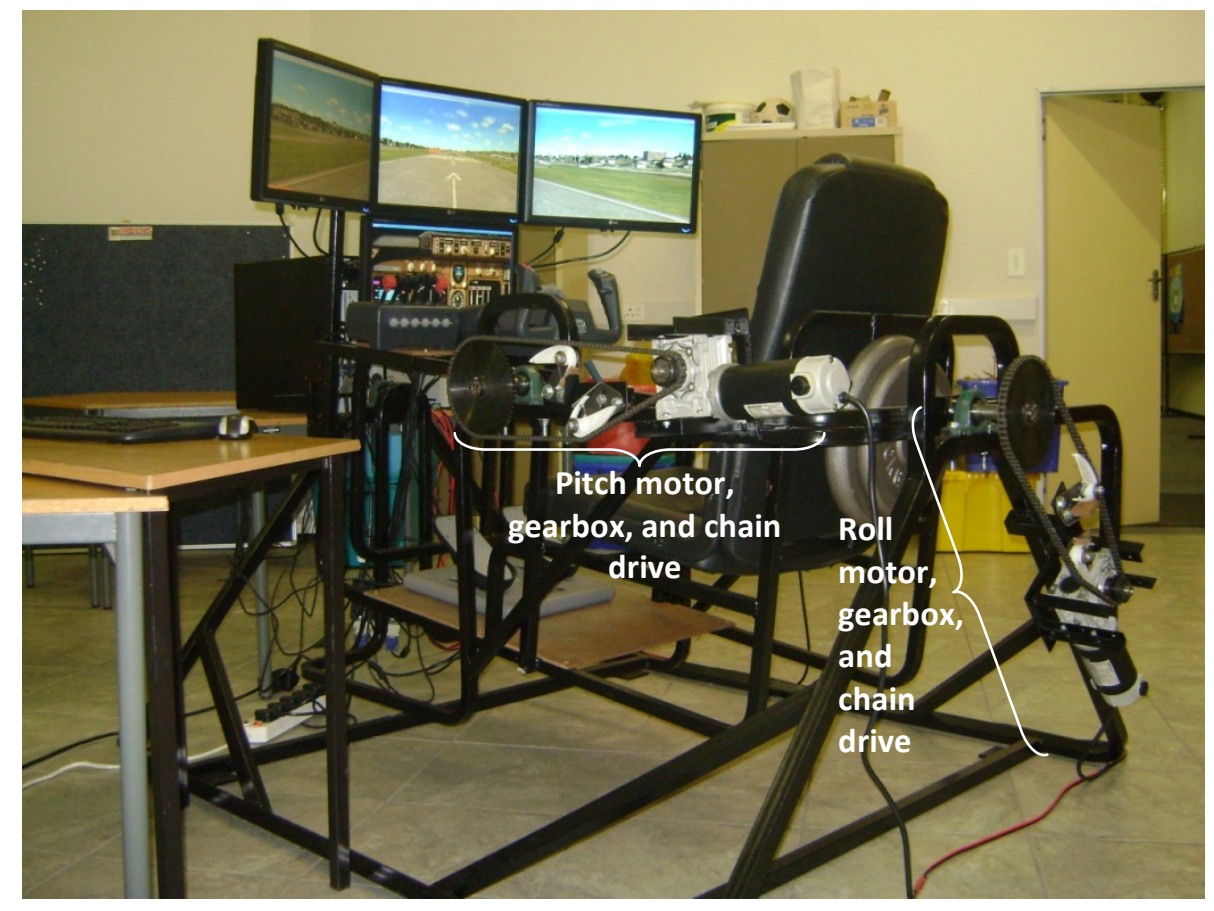

Fig. 5 The completed simulator, showing electrical drives for both pitch-and roll-frames (with safety covers removed)

To validate the design, the flight simulator was subjected to a physical test to determine the actual maximum torque output from the motors. This was calculated by monitoring the maximum electrical current drawn by the motors, and then reading the corresponding torque values from the motor manufacturer's curves [26]. The current never exceeded 4 A, which was equivalent to a motor output torque of 0.09 N.m. Therefore, the maximum torque provided to the platform shafts was (this was for the pitch platform):

$$
T_{\max }=T_{\text {motor }} n_{r, \text { total }} \eta_{\text {gearbox }}=(0.09)(335)(0.53)=15.98 \mathrm{~N} . \mathrm{m}
$$


This was considerably lower than the design torque of 90 N.m. This showed that the platform was very well balanced and that there is adequate extra torque available for when the occupant moves forward and causes a reduced offset of his CG with respect to the axis of rotation, as discussed earlier. It was therefore concluded that the motors would not be overloaded during full-load operation.

The flight simulator responds well to user inputs, especially considering the fact that it is a two degrees-of-freedom simulator. A retired airliner commander with nearly 30,000 logged flying hours flew the simulator, and found that it realistically simulates the 'feel' of a large commercial airliner [29].

\section{Educational value of the simulator}

The simulator was originally intended to be displayed (and operated) in a permanent aviation exhibit at SciEnza, the science eploratorium of the University of Pretoria. The purpose was to teach children, students and the general public more about flight simulators and aviation in general. It was also done to promote mechanical and aeronautical engineering among the centre visitors.

Although the aforementioned is still envisioned, a second, more important, educational purpose was identified. This entails using the simulator in mechanical and aeronautical engineering training at the University of Pretoria. Specific applications have not yet been identified, but the system might be used in practical work in undergraduate subjects such as Dynamics, Vibration, Electrotechnics, and Control. Furthermore, it can also be used as a research platform for final-year design and dissertation projects, as well as for advanced research during post-graduate studies. 
Finally, electrical-, electronic- and computer engineering students might also benefit, as custom software and hardware can be written and designed for- and tested on this system.

\section{Conclusion}

An educational flight simulator was designed, built and tested using commercial flight simulation software (Microsoft Flight Simulator $X$ ) to simulate the motion of a large airliner (such as a Boeing 777). It has been shown that a two degrees-of-freedom (pitch and roll) motion platform can be effectively realized by using interlocking pitch- and roll-frames (with total rotating mass of $200 \mathrm{~kg}$ ), each actuated by a $180 \mathrm{~W}$ brushed DC motor.

To ensure adequate output torque (and speed), the DC motors were connected to commercial gearbox and chain transmissions providing an overall speed reduction of 335:1. The measured torque (16 N.m) was safely and conservatively provided for by the DC drive at 101 N.m. The total system cost (hardware and software) was US\$7,410, proving that an affordable flight simulation solution was available for educational purposes.

The simulator is to be displayed at SciEnza, the science exploratorium of the University of Pretoria to promote aviation as well as mechanical and aeronautical engineering, among the general public, children and students. It is also intended to be used by the School of Engineering in engineering education by acting as a tool to be used in undergraduate practical work, as well as a research platform for final-year and post-graduate students.

\section{Acknowledgements}

The authors would like to thank the following persons and organisations for their invaluable assistance during the development of this flight simulator: the National Aerospace Centre for funding the project; Ian Hopper (BFF Design Ltd) for providing design advice and for 
proofreading the manuscript; Microsoft, and especially their representative, John Press, who granted the authors permission to use Flight Simulator $X$ in a science exploratorium, as well as donating two copies of the software; the members of Project OpenSky, especially Philip Foglar, who granted the authors permission to use their fantastic Boeing 777 for the simulation model; Brig. Gen. Desmond Barker (SAAF retd.), Mr. Steve Haselum, and Mr. Hein Swart (Centre for Scientific and Industrial Research, Pretoria); Prof. Nico Theron, Prof. Schalk Els, Prof. Stephan Heyns, Riaan Meeser and George Breytenbach from the Department of Mechanical and Aeronautical Engineering, University of Pretoria; and Dr. Michiel Heyns (Investmech).

\section{References}

[1] G. Tarantino, C. Fazio, and R. M. Sperandeo-Mineo, 'A pedagogical flight simulator for longitudinal airplane flight', Computer Applications in Engineering Education 18(1) (2010), 144-156.

[2] D. B. Landrum, J. Cerny, L. Warden, and A. Meyer, Affordable flight simulation in an educational environment, Proceedings of the $63^{\text {rd }}$ Annual Forum of the American Helicopter Society 3 (2007), 2241-2251.

[3] M. G. Perhinschi, M. R. Napolitano, Flight dynamics and control education enhancement using a motion based flight simulator, Proceedings of the Spring 2007 American Society of Mechanical Engineering Education North Central Section Conference (West Virginia Institute of Technology, 30-31 March 2007).

[4] R. S. Burns, M. W. Duquette, J. B. Howerton, and R. J. Simko, Development of a lowcost simulator for demonstration and engineer training, Report no. AFRL-VA-WP-TP2003-317, Air Vehicles Directorate, Air Force Research Laboratory (Ohio, USA, 2003). 
[5] E. J. Jumper, and J. W. Baughn, 'The use of Microsoft Flight Simulator in aerospace education', American Institute of Aeronautics and Astronautics 127 (1991), 875-883.

[6] Z. Lei, J. Hongzhou, and L. Hon, PC Based High Quality and Low Cost Flight Simulator, Proceedings of the IEEE International Conference on Automation and Logistics Proceedings (Jinan, China August 18 - 21, 2007).

[7] R. Hosman, S. Advani, and N. Haeck, Integrated design of flight simulator motion cueing systems, Proceedings of the Royal Aeronautical Society Conference on Flight Simulation (London, UK, May 2002).

[8] D. J. Allerton, A distributed approach to the design of a real-time engineering flight simulator, Proceedings of the $21^{\text {st }}$ International Council of the Aeronautical Sciences (ICAS) Congress, paper ICAS-98-1,1,2 (Melbourne, Australia, 13-18 Sept. 1998).

[9] J. A. Boldovici, Simulator Motion, Technical Report 961: AD-A257 683, United States Army Research Institute for the Behavioral and Social Sciences (Washington, DC, Sept. 1992).

[10] L. R. Ribeiro, and N. M. F. Oliviera, UAV autopilot controllers test platform using Matlab/Simulink and X-Plane, Proceedings of the $40^{\text {th }}$ ASEE/IEEE Frontiers in Education Conference (Washington, DC, 27 - 30 Oct. 2010).

[11] F. Holtzapfel, I. Sturhan, and G. Sachs, Low-cost PC based flight simulator for education and research, Proceedings of the AIAA Modeling and Simulation Technologies Conference and Exhibit (Monterey, California, 5-8 Aug. 2002).

[12] P. R. Grant, and L. D. Reid, 'Motion washout filter tuning: rules and requirements', Journal of Aircraft 34(2) (1997), 145-161.

[13] S. H. Koekebakker, Model based control of a flight simulator motion system, PhD thesis, Delft University of Technology (Delft, The Netherlands, 2001). 
[14] M. A. Nahon, and L. D. Reid, 'Simulator motion-drive algorithms: a designer's perspective', Journal of Guidance 13(2) (1990), 356-362.

[15] Built For Fun, http://buggies.builtforfun.co.uk/Sim/ (Accessed 18 February 2009).

[16] D. Barker (Brig. Gen. (South African Air Force, retd.)), Personal communication, CSIR, (Pretoria, South Africa, 17 March 2009).

[17] H. Lenferink, Building your own flight simulator cockpit. http://www.fsinsider.com/freeflight/Pages/BuildingASimulatorCockpit.aspx (Accessed 20 March 2009).

[18] C. T. E. Lan, J. Roskam, Airplane Aerodynamics and Performance (DARCorporation, Lawrence, Kansas, USA, 2003).

[19] H. M. Reynolds, The inertial properties of the body and its segments (University of Michigan, Michigan, 1985).

[20] M. R. Macedonia, and P. S. Rosenbloom, Entertainment Technology and Virtual Environments for Training and Education, in The Internet and the University: 2000 Forum. Eds. R. Larson, J. Meyerson, and M. Devlin (Educause, Boulder, Co, USA, pp. 79-95, 2001).

[21] Robot Electronics: MD03 Technical Documentation, http://www.robotelectronics.co.uk/htm/md03tech.htm (Accessed 20 July 2009).

[22] D. Halliday, R. Resnick, and J. Walker, Fundamentals of Physics (7 $7^{\text {th }}$ Edition) (John Wiley \& Sons, Inc., New York, 2005).

[23] M. McLafferty, Boeing Commercial Airplanes, e-mail communication. 19 August.

[24] J. H. Blakelock, Automatic control of aircraft and missiles (Wiley Interscience Publications, New York, 1991).

[25] SimMartket, http://secure.simmarket.com/pete-dowson-fsuipc3.phtml (Accessed 10 April 2011). 
[26] Transtecno, Permanent Magnet DC Electric Motors EC (catalogue and technical documentation):

http://www.mht.lt/cms/uploaded//Transtecno/katalogai/P_EC_ott08_.pdf (Accessed 20 May 2009).

[27] Communica,

http://www.communica.co.za/ProductDetails.aspx?DataLink=P_001273476725

(Accessed 10 April 2011).

[28] CH Products, Flight Simulator Controls. http://www.chproducts.com/ (Accessed 20 February 2011).

[29] D. Velleman, Flight simulator testing by Capt. (SA Airways, retd.) Douglas Velleman (Pretoria, South Africa, 11 March 2011). 